\section{Controlled Pulsatile Drug Release from a Ferrogel by a High-Frequency Magnetic Field}

\author{
Shang-Hsiu Hu, Ting-Yu Liu, Dean-Mo Liu,* and \\ San-Yuan Chen*
}

Department of Materials Sciences and Engineering, National Chiao Tung University, Hsinchu, Taiwan, 300, ROC

Received March 30, 2007

Revised Manuscript Received July 31, 2007

Stimuli-responsive controlled release drug from polymeric devices has been received great attention, as it provides advantages such as better delivery efficiency and site-specific therapy over the conventional routes of drug delivery. By making use of these advantages, a number of studies have been successfully proposed to integrate active drug molecules and host materials, to manipulate drug release desirably. Many previous studies have been reported in response to specific stimuli, such as temperature, ${ }^{1-3} \mathrm{pH},{ }^{4,5}$ electric field, ${ }^{6,7}$ mechanical signal, ${ }^{8}$ and ultrasound. ${ }^{9}$ A very recent achievement of a protein-containing hydrogel, reported by Ehrick et al., ${ }^{10}$ addressed the stimuli-sensitive hydrogel that can be triggered as a result of the conformational change of the embedded calmodulin protein interacting to bioactive agents. Such a transfer from sensing actuation to mechanical action is novel, but it may be hard for it to reach high accuracy and sensitivity in therapeutic dosing as a burst-controlled drug delivery system. The use of a magnetic field to modulate drug release from polymeric matrices was previously developed. ${ }^{11-13}$ Saslawski et al. reported the alginated microspheres for pulsed release of insulin by an oscillating magnetic field. ${ }^{14}$ The release rate of insulin from alginate-strontium ferrite microspheres was enhanced in the absence of a magnetic field. Recently, a polyelectrolyte microcapsule embedded with $\mathrm{Co} / \mathrm{Au}$ used external magnetic fields of $100-300 \mathrm{~Hz}$ and 1200 Oe to increase its permeability to macromolecules. ${ }^{15}$ In previous studies, a ferrogel with direct current (dc) magnetic-sensitive properties has been characterized and the amount of drug released from the ferrogel was effectively restricted while applying an external dc magnetic field. ${ }^{16,17}$ So far, little investigation has been addressed on the controlled drug release under high-frequency magnetic fields (HFMF). A real-time burst release of drug needs a fast-responsive drug release system to "inject" a precise dose of drug when the body needs it and to "stop" or "slow" the release right after the injection; however, it is hard to easily achieve this with traditional stimuli-responsive hydrogels. A controllable pulsatile-type drug delivery device with repeatable dosing ability in therapeutically effective precision is clinically desirable. Therefore, in this communication, a high-frequency magnetic field (HFMF) triggered pulsatile drug delivery ferrogel with a mechanically reliable and flexible hybrid structure composed of gelatin and magnetic nanoparticles (nanomagnets) of $10-250 \mathrm{~nm}$ in diameter is reported. Furthermore, under cyclic exposures to the high-frequency magnetic stimuli, a highly controllable and repeatable burst release with desirable precision from the ferrogels is achieved.

* Corresponding authors. Telephone: +886-3-5731818. Fax: +886-35725490; E-mail: (S.-Y.C.) sanyuanchen@mail.nctu.edu.tw; (D.-M.L.) deanmo_liu@yahoo.ca.

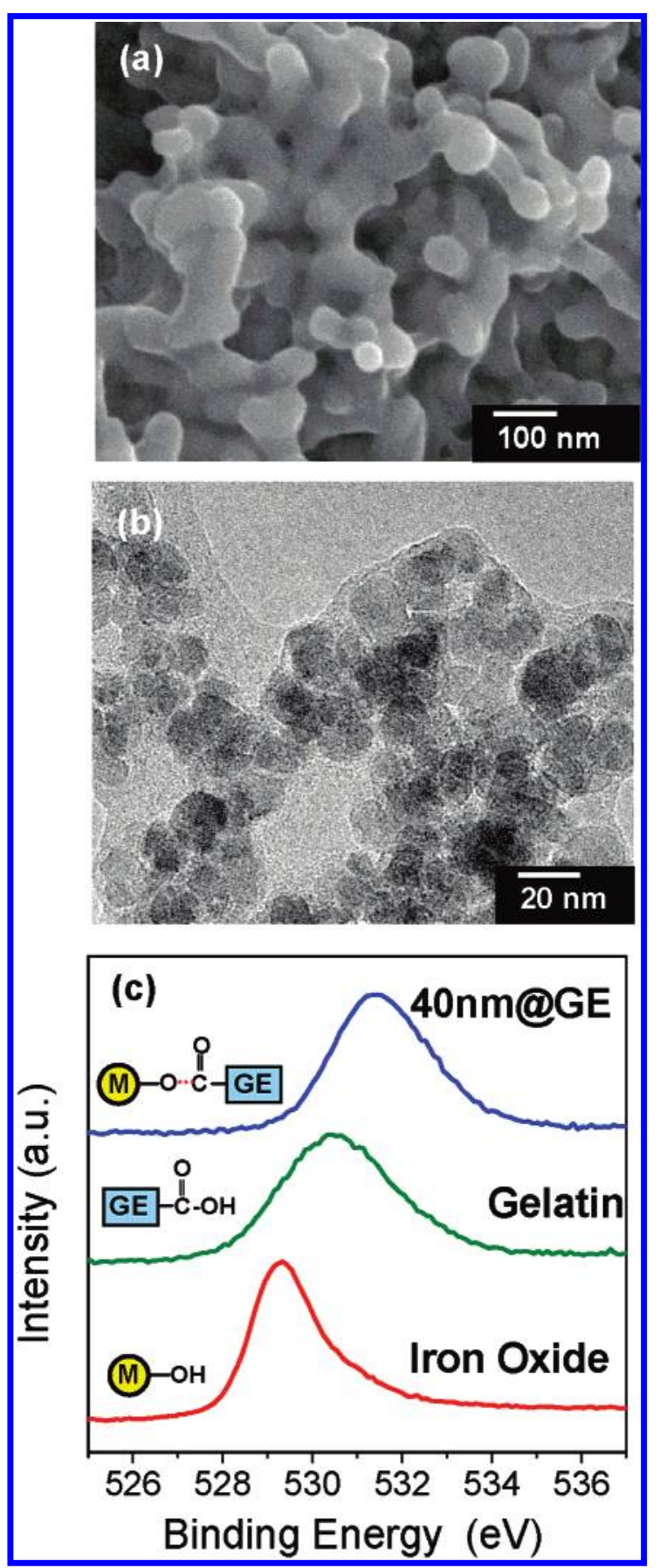

Figure 1. (a) SEM, (b) TEM, and (c) XPS analyses of ferrogels composed of iron oxide nanoparticles and a gelatin matrix.

Figure 1a illustrates the SEM images of one of the ferrogels shaping in cylindrical geometry, namely 40nm@GE ferrogel, where 40nm@GE represents the ferrogels with gelatin crosslinked with genipin, reinforced by $2 \mathrm{wt} \%$ chitosan, and $3 \%$ $\mathrm{Fe}_{3} \mathrm{O}_{4}$ magnetic nanoparticles (nanomagnets) of average $40 \mathrm{~nm}$ in diameter. Chitosan provides several functions in the final hybrid structure, such as (1) matrix for drug encapsulation, (2) materials to provide deformation motion. and (3) binder to link the nanoparticles. As shown, the iron nanoparticles were embedded into the polymer matrix and the nanomagnets displayed a uniform spherical size and dispersed well in the ferrogel. TEM image, i.e., Figure 1b, exhibits that the gelatin 

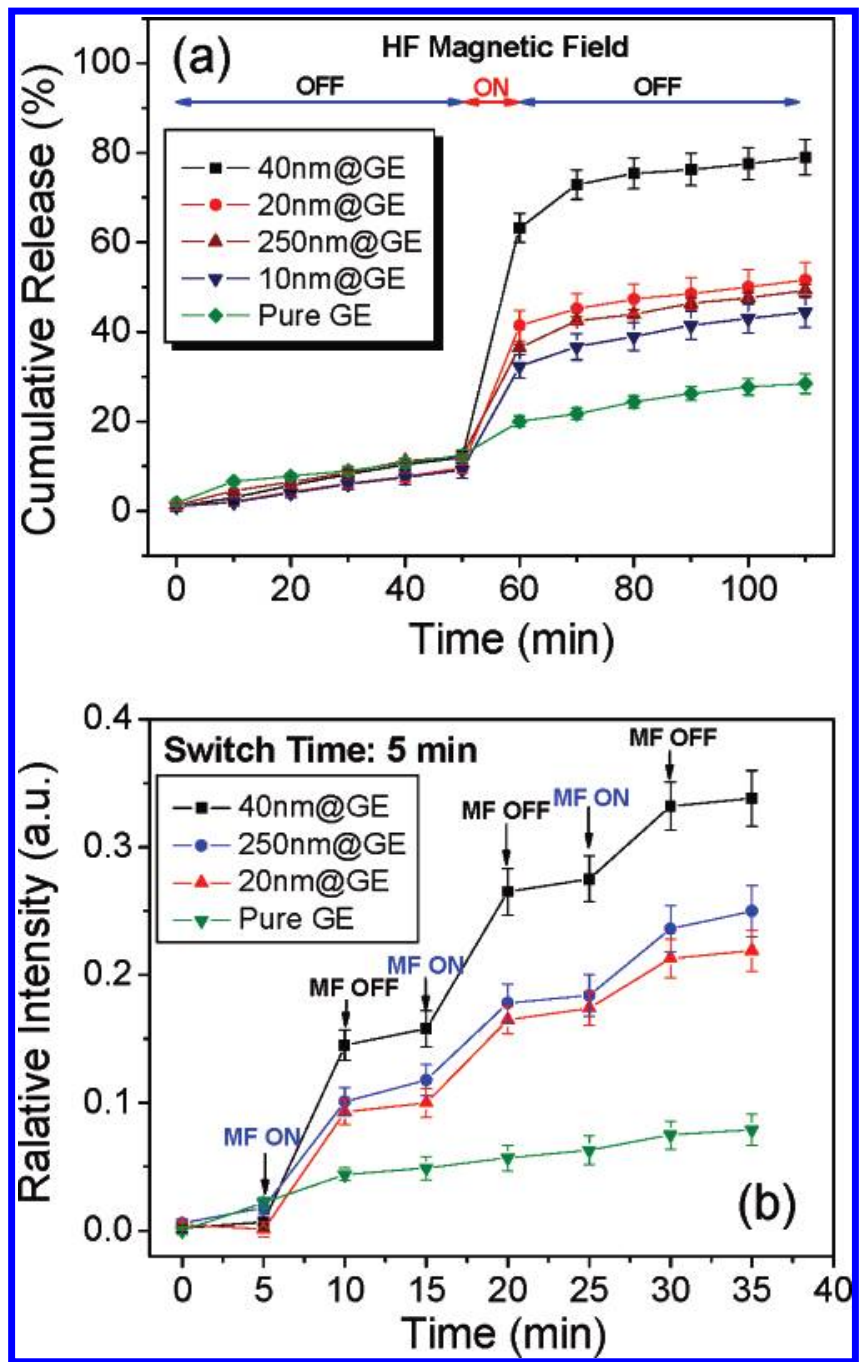

Figure 2. (a) Drug release behaviors of the ferrogels under a $10 \mathrm{~min}$ exposure of high-frequency magnetic field (HFMF) and (b) the onoff switch operations of high-frequency magnetic field (HFMF) manipulating the cumulative drug release of the ferrogels with different sizes of the nanomagnets.

was intimately attached to the surface of the nanomagnets, and the nanomagnet particles also percolated as a continuous phase, indicating the resulting ferrogels are virtually an interpenetrating network composites. This also suggests that the physical or thermal interactions between these two closely contact, interpenetrating phases can be easily triggered and accessible from one phase to the other at a nanometric level. This also ensures fast-responsive properties of the ferrogels that can be achieved as disclosed in the forthcoming analysis.

The nanomagnets were essentially chemically bounded with the gelatin matrix to form the ferrogel, which further evidenced from XPS analysis, in Figure 1c. The binding energy of $\mathrm{O}_{1 \mathrm{~s}}$ is detected from 527.0 to $537.0 \mathrm{eV}$, and the peak of iron oxide was at $529.3 \mathrm{eV}$, which is reasonably consistent with literature report, i.e., $528-531 \mathrm{eV}$. A peak at $530.4 \mathrm{eV}$ for the gelatin indicates its carboxylic acid group; however, a shifting to higher energy region $(531.4 \mathrm{eV})$ when the iron oxide particles were incorporated, implying that the carboxylic acid was chemically bonds or immobilized, onto the nanomagnet surface. The higher binding energy is believed to be a result of esterification between hydroxide moiety on the surface of the MNPs and carboxylic acid group of gelatin, forming a $\mathrm{COO}-\mathrm{Fe}$ bonding, indicating an excellent chemical affinity between both participating phases and a mechanically strong solid network of the ferrogels is achieved.

Upon magnetic manipulation, the drug (vitamin $\mathrm{B}_{12}$ ) released from the ferrogels was demonstrated in Figure $2 \mathrm{a}$. The ferrogels with different particle sizes of the nanomagnets showed different release rates even though they possessed the same concentration of the nanomagnets. In comparison, pure polymer gelatin showed faster drug release rate than that of the ferrogels, indicating the nanoparticles are acting as physical barriers to inhibiting the diffusion of the drug molecules. After the initial time period (50 min) of slow release, the high-frequency magnetic field (HFMF) was applied to the ferrogels for $10 \mathrm{~min}$, and then was removed. The amount of drug release from the 40nm@GE ferrogel burst to a level as high as about 63\%, compared to $12 \%$ during the first 50 -min release in the absence of the HFMF. The finding strongly indicates that the applied HFMF accelerated considerably the drug release from the ferrogels of 40nm@GE composition by as large as 5 times. More specifically, the amounts of drug release from the ferrogels with different particle size were increased in the order of 40nm@GE $>$ 20nm@GE > 250nm@GE > 10nm@GE > pure gelatin under the same magnetic stimulus. However, the release profiles after the 10-min burst, restored almost completely right after removal of the high-frequency magnetic stimulus, and behaved almost exactly the same as those at the initial 50-min period. A real-time, fast-responsive drug delivery ferrogels can be achieved and the faster responsivity of the ferrogels with respect to the HFMF stimulus offers greater potential for an in-time drug release device for medical applications.

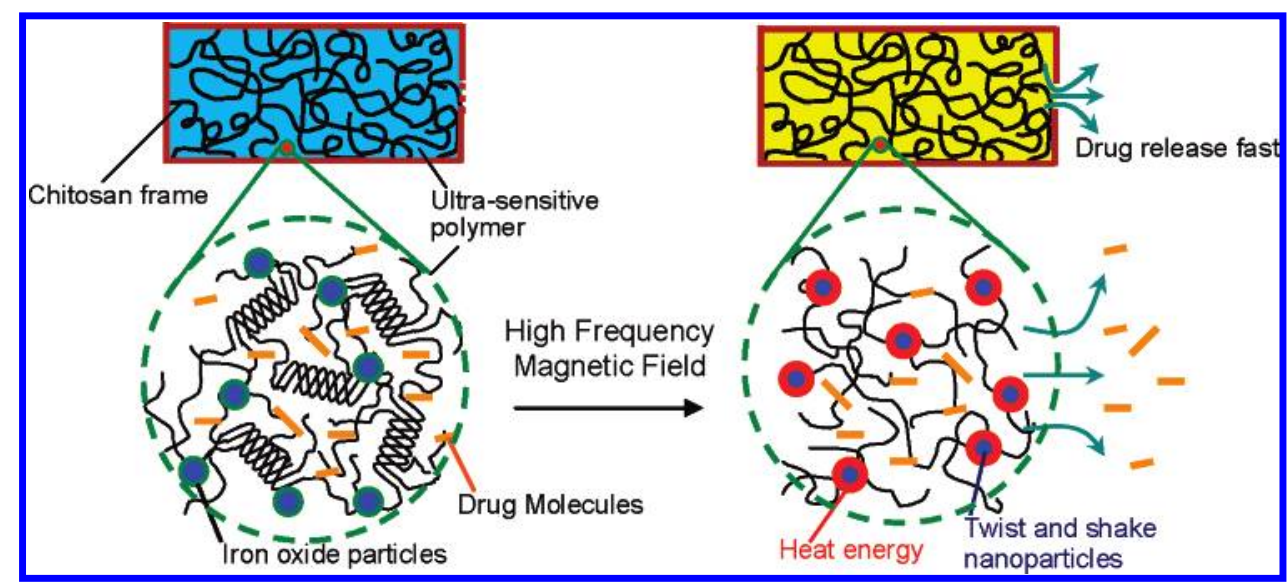

Figure 3. Schematic drawing of structures of ferrogel which exhibited a triple-helix structure to restrict the drug molecules to release. While applying the high-frequency magnetic field (HFMF), the magnetic nanoparticles provides the heat energy to release the structures and twist and shake the polymer molecular chains to effectively accelerate drug release rate. 


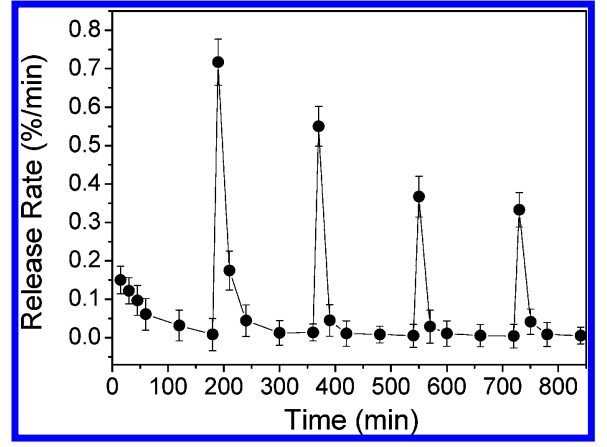

Figure 4. Cyclic drug release rates of 40nm@GE ferrogels under a 5-min period of HFMF stimuli and a long $180 \mathrm{~min}$ switching duration, where a longer switching time period ensures the drug to reach a kinetically favorable distribution in the ferrogel for a subsequent burst release.

Upon tripled on-off operations of the HFMF to the ferrogels, Figure $2 b$ shows that reproducible slow-to-burst release profiles while consecutively applying the magnetic stimulus at a 5-min switching time period and the release profile restored immediately when the stimulus was removed. The findings imply two important characteristics underlying the drug release pattern of the high-frequency-induced ferrogels: first, under a short time "on-off" exposure of the magnetic stimulus, the ferrogels followed exactly the signals switching from burst to slow release of the drug instantly, and second, the amounts of drug bursting from the ferrogels maintained accurate dosing under cyclic onoff operations of the HFMF. The latter suggests the ferrogels prepared deformed elastically and showed potential antifatigue behavior, while the former indicates a fast-acting response of the ferrogels that can be technically achieved with desirable stimuli-sensitive control of drug delivery through the incorporation of $40 \mathrm{~nm}$ nanomagnets (average diameter).

The bursting release of the drug from the ferrogels is indicative of a mixture of mechanical actions imposed by the ferrogels, which may include (1) an "open" configuration of the network structure and (2) an elastic deformation (i.e., contractile deformation) of the ferrogels, while being subject instantly to the HFMF stimulus. Under HFMF, the nanomagnets were activated kinetically and possibly thermally (for larger nanoparticles), and they transformed the structural or molecular configuration of the ferrogels upon microstructural deformation (shrinking) of the gelatin matrix. Gelatin is considered to be a thermosensitive polymer since its conformation is a coil in the warm water $(>40$ ${ }^{\circ} \mathrm{C}$ ) but turns into a gel structure involving triple-helix sequences on cooling. ${ }^{18,19}$ Among the ferrogels prepared, magnetization evaluation (see Supporting Information, Figure S1) showed 40nm@GE composition exhibiting the broadest hysteresis loop compared to the others, indicating more energy can be generated instantly under the high-frequency magnetic stimulus. In other words, the 40nm@GE ferrogel was effectively thermally activated, where a rapid change in molecular conformation of the gelatin matrix from denser to looser configuration, as schematically illustrated in Figure 3, is expected in the ferrogels by a high-frequency magnetically induced thermal variation near the nanoenvironment surrounding the nanomagnets.

For a long HFMF switching duration, i.e., $180 \mathrm{~min}$, Figure 4 illustrates a series of bursts started after a first 3-h slow release, under a 5-min duration of HFMF stimuli, where a gradual reduction in the burst release rate was detected with time. Even though the longer switching time period of the high-frequency magnetic stimulus ensures a kinetically favorable drug migration, the insufficient drug amount in the ferrogel can hardly sustain the same burst release rate. However, a relatively slow to nearzero release rate was detected immediately after removal of the HFMF and kept relatively constant during the time duration, i.e., $180 \mathrm{~min}$, between each stimulus. The same scenario of burst and slow release profiles repeated accurately for a time period of $840 \mathrm{~min}$ of drug release operation proved that the ferrogels exhibited an excellent elastic deformation property. This allows consecutive operation for a continuously controlled burst release of a drug into a patient's body to maintain a therapeutically effective dose in the body for a longer period of time to efficiently treat the disease locally or systemically. One clinically important advantage of this high-frequency-modulated fastacting drug delivery ferrogel over other existing drug delivery devices is that it can potentially meet immediate urgent physiological needs and, with dosing, compatibly match chronological changes in patients' bodies.

Supporting Information Available: Text giving experimental details and effect of magnetic partical size with a a figure showing the latter. This material is available free of charge via the Internet at http://pubs.acs.org.

\section{References and Notes}

(1) Zhang, X. Z.; Wu, D. Q.; Chu, C. C. Biomaterials 2004, 25, 37933805 .

(2) Eeckman, F.; Moës, A. J.; Amighi, K. Int. J. Pharm. 2004, 273, 109119.

(3) Hu, Z.; Xia, X. Adv. Mater. 2004, 16, 305-309.

(4) Chen, S. C.; Wu, Y. C.; Mi, F. L.; Lin, Y. H.; Yu, L. C.; Sung, H. W. J. Controlled Release 2004, 96, 285-300.

(5) Das, M.; Mardyani, S.; Chan, W. C. W.; Kumacheva, E. Adv. Mater 2006, $18,80-83$.

(6) Murdan, S. J. Controlled Release 2003, 92, 1-17.

(7) Abidian, M. R.; Kim, D. H.; Martin, D. C. Adv. Mater. 2006, 18, 405-409.

(8) Lee, K. Y.; Peters, M. C.; Mooney, D. J. Adv. Mater. 2001, 13, 837839.

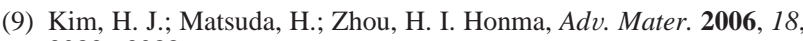
3083-3088.

(10) Eheick, J. D.; Deo, S. K.; Browning, T. W.; Bachas, L. G.; Madou, M. J.; Daunert, S. Nat. Mater. 2005, 4, 298-302.

(11) Kost, J.; Wolfrum, J.; Langer, R. J. Biomed. Mater. Res. 1987, 21 , $1367-1373$.

(12) Edelman, E. R.; Kost, J.; Bobeck, H.; Langer, R. J. Biomed. Mater. Res. 1985, 19, 67-83.

(13) Kost, J.; Noecker, R.; Kunica, E.; Langer, R. J. Biomed. Mater. Res 1985, 19, 935-940.

(14) Saslawski, O.; Weingarten, C.; Benoit, J. P.; Couvreur, P. Life Sci. 1988, 42, 1521-1528.

(15) Lu, Z.; Prouty, M. D.; Guo, Z.; Golub, V. O.; Kumar, C. S. S. R.; Lvov, Y. M. Langmuir 2005, 21, 2042-2050.

(16) Liu, T. Y.; Hu, S. H.; Liu, T. Y.; Liu, D. M.; Chen, S. Y. Langmuir 2006, 22, 5974-5978.

(17) Liu, T. Y.; Hu, S. H.; Liu, K. H.; Liu, D. M.; Chen, S. Y. J. Magn. Magn. Mater. 2006, 304, e397-e399.

(18) Crescenzi, V.; Francescangeli, A.; Taglienti, A. Biomacromolecules 2002, 3, 1384-1391.

(19) Bulcke, A. I. V. D.; Bogdanov, B.; Rooze, N. D.; Schacht, E. H.; Cornelissen, M.; Berghmans, H. Biomacromolecules 2000, 1, 31-38.

MA0707584 